\title{
Erratum: Effects of Individual Education Using a Treating-to-target Strategy in Patients with Rheumatoid Arthritis
}

\author{
Seung In Paek ${ }^{1}$, Seung Min Jung ${ }^{2}$, Jennifer Lee ${ }^{3}$, Seung-Ki Kwok ${ }^{3}$, Wan-Uk Kim³ ${ }^{3}$ Sung-Hwan Park ${ }^{3}$, Ji Hyeon Ju ${ }^{3}$, \\ Kyeong-Yae Sohng ${ }^{4}$ \\ ${ }^{1}$ Department of Internal Medicine, Seoul St. Mary's Hospital, The Catholic University of Korea, ${ }^{2}$ Division of Rheumatology, Department of \\ Internal Medicine, Yonsei University College of Medicine, ${ }^{3}$ Division of Rheumatology, Department of Internal Medicine, Seoul St. Mary's \\ Hospital, College of Medicine, The Catholic University of Korea, ${ }^{4}$ Department of Nursing, College of Nursing, The Catholic University of Korea, \\ Seoul, Korea
}

J Rheum Dis 2018;25:255-262

https://doi.org/10.4078/jrd.2018.25.4.255

The original version of this article contained an error of the ORCID identifier of corresponding author (Ji Hyeon Ju).

The ORCID identifier of corresponding author is https://orcid.org/0000-0002-1381-5466.

The original version has been already revised in the journal's online site.

We apologize for this error. 